\title{
A Torque Cancelling System for Quick-Motion Robots
}

\author{
Daigoro Isobe \\ University of Tsukuba \\ Japan
}

\section{Introduction}

A new torque cancelling system that stabilizes mechanical sway in quick-motion robots is discussed in this chapter. Mechanical sway generated by reaction moments occurring in the motion of, for example, space stations is an important issue that cannot be neglected. Motion controls using the conservative law of angular momentum are applied by driving control momentum gyros (CMG) (Ookami et al., 2008) or reaction wheels (Yoshida et al., 2002). Applications of the gyros and the wheels are generally limited to slow momentum transitions relying upon high-gain feedback control. Although the same strategy can be used in the motion control of robots when the momentum transitions are slower, the dynamics of the robots should be considered to effectively control quicker motions. The torque cancelling system (TCS) discussed in this chapter cancels the reaction moment generated by the motion of an object by considering the precise dynamics of the object and the body of the robot itself. The reaction moment can be obtained accurately using a newly proposed solution scheme of inverse dynamics that handles the dynamics of complex robotic architectures by modelling them with finite elements. Once the reaction moment is known, it can be cancelled by applying an anti-torque to a torque-generating device.

The newly proposed solution scheme of inverse dynamics mentioned above is called the parallel solution scheme (Isobe et al., 2000; Isobe, 2004), and it was developed on the basis of a finite element approach. In contrast to conventional and revised schemes that use dynamic equations (for example: Nakamura \& Ghodoussi, 1989; Nakamura, \& Yamane 2000; Sugimoto, 2003), the proposed scheme can handle different types of configurations, such as open-loop, closed-loop, or multibranch link systems, and can also consider the elasticity of constituted links or passive joints by only changing the input numerical model, without the need to revise any part of the scheme. Its validity in various feedforward control experiments of various kinds of link systems has been verified (Isobe et al., 2003; Isobe et al., 2006; Isobe \& Kato, 2008).

In this chapter, an outline of the parallel solution scheme is first described along with some numerical examples of flexible and underactuated models. Then, some examples of torque cancelling resulting from accurate calculations of dynamics are demonstrated by showing the experimental results carried out on a simple rotor-TCS system. The systems used for the rotor part include rigid and flexible outboard and inboard rotors, where difficult assumptions are normally required to consider the dynamics accurately. A numerical example of a complex robotic architecture is also presented to verify the use of the system. 


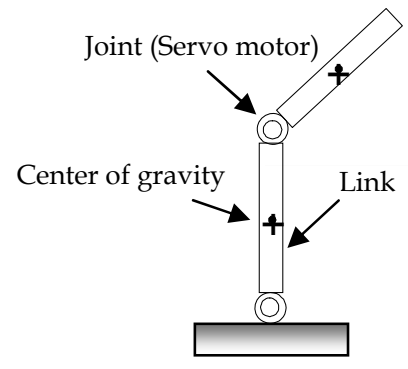

(a) Link system

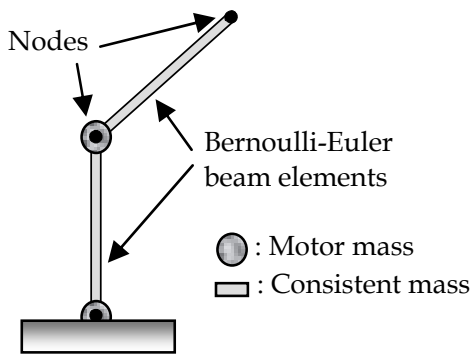

(b) Finite element modelling with consistent mass distribution

Fig. 1. Modelling of link systems using finite elements

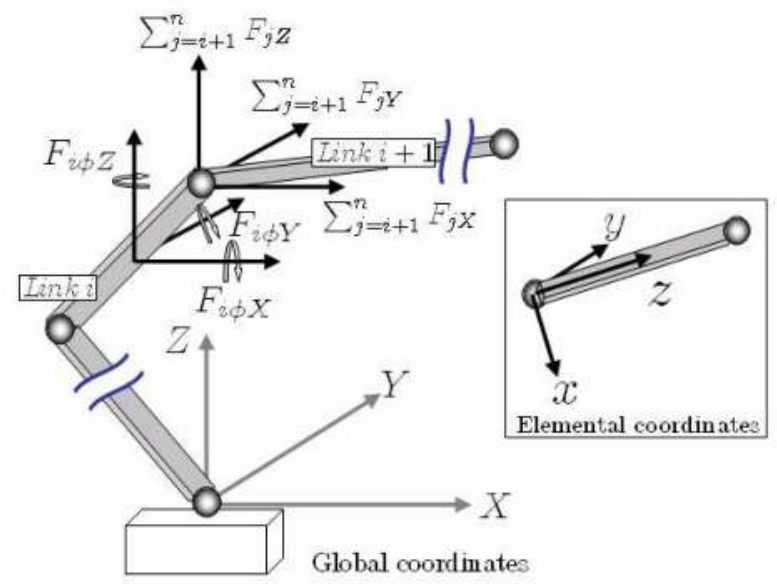

Fig. 2. Nodal forces acting on the $i$ th link in an $n$-link system with consistent mass distribution

\section{Parallel solution scheme of inverse dynamics}

\subsection{Calculation of joint torques}

In the parallel solution scheme, a link system consisting of motor joints and links, as shown in Fig. 1(a), is modelled and subdivided using finite elements. A link is substituted with a single Bernoulli-Euler beam element in the revised version, as shown in Fig. 1(b), assuming a consistent mass distribution along the link (Isobe \& Kato, 2008). The consistent mass matrix of a beam element is formulated in the same manner as the displacement function and does not require an expression of the centre of gravity. This type of modelling has the merits of reducing computational time without lowering accuracy, particularly in cases of occurrence of elastic deformations. It requires only one element subdivision per member for cases of infinitesimal deformation because the deformation of the element is defined using a high-order displacement function.

Figure 2 shows the nodal forces based on global coordinates acting on the $i$ th link in a threedimensional open-loop $n$-link system with a consistent mass distribution. The joint torque $\tau_{i x}$ 
required around the $x$-elemental axis on the $i$ th link is determined by adding the $i+1$ th joint torque $\tau_{(i+1) \times}$ to the sum of the moments of inertia acting on this link and is expressed by the nodal forces based on elemental (or link) coordinates as

$$
\tau_{i x}=l_{i}\left(\sum_{j=i+1}^{n} F_{j}\right)_{y}+F_{i \phi x}+\tau_{(i+1) x},(i, j=1 \sim n),
$$

where $l$ is the link length and $F$ is the nodal force. $\sum F$ indicates the resultant force, the subscripts $i, j$ the link number, $x, y, z$ the elemental coordinate components, $X, Y, Z$ the global coordinate components, and $\varphi$ the angular components. The right hand side of Eq. (1) becomes different in a scheme with a lumped mass distributed at the centre of gravity (Isobe, 2004). By considering the other components around the $y$ - and $z$-axes and arranging them into global coordinates $(X, Y, Z)$ in matrix form, the joint torque vector is expressed as

$$
\left\{\tau^{n}\right\}=\left[L^{n}\right]\left[T^{n}\right]\left\{P^{n}\right\},
$$

where $\left\{P^{n}\right\}$ is a $6 n \times 1$ vector related to the nodal force and is defined as

$$
\left\{P^{n}\right\}=\left\{\begin{array}{c}
P_{1} \\
P_{2} \\
\bullet \\
\bullet \\
P_{n}
\end{array}\right\} \text {, where }\left\{P_{i}\right\}=\left\{\begin{array}{c}
\sum_{j=i+1}^{n} F_{j X} \\
\sum_{j=i+1}^{n} F_{j Y} \\
\sum_{j=i+1}^{n} F_{j \mathrm{Z}} \\
F_{i \phi X} \\
F_{i \phi Y} \\
F_{i \phi \mathrm{Z}}
\end{array}\right\} .
$$

$\left[T^{n}\right]$ is a $6 n \times 6 n$ transformation matrix and is defined as

$$
\left[T^{n}\right]=\left[h^{n}\right]\left[T_{G E}^{n}\right],
$$

where $\left[h^{n}\right]$ is a correction matrix between the $x-y$ and $z-x$ coordinate systems, which simply inverts the signs of the components in the $y$-axis direction. $\left[T^{n} G E\right]$ is a transformation matrix between the global and elemental coordinates and is expressed as

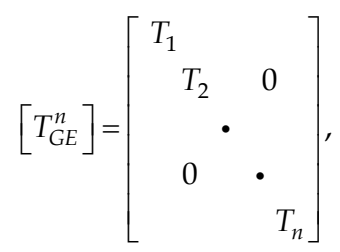

where 


$$
\left[T_{i}\right]=\left[\begin{array}{cc}
A_{i} & 0 \\
0 & A_{i}
\end{array}\right]
$$

and

$$
\left[A_{i}\right]=\left[\begin{array}{ccc}
\cos \phi_{i X x} & \cos \phi_{i Y x} & \cos \phi_{i Z x} \\
\cos \phi_{i X y} & \cos \phi_{i Y y} & \cos \phi_{i Z y} \\
\cos \phi_{i Z x} & \cos \phi_{i Z y} & \cos \phi_{i Z z}
\end{array}\right],
$$

where $\varphi_{i X x}$, for example, represents the rotational angle between the $X$ (global) and $x$ (elemental) coordinates.

$\left[L^{n}\right]$ in Eq. (2) is a $3 n \times 6 n$ matrix related to link length and is expressed as

$$
\left[L^{n}\right]=\left[T_{\Lambda}^{n}\right]\left[\Lambda^{n}\right]
$$

where $\left[T^{n} \Lambda\right.$ is a transformation matrix between each elemental coordinate and is expressed as

$$
\left[T_{\Lambda}^{n}\right]=\left[\begin{array}{ccccccc}
T_{11} & T_{12} & T_{13} & \bullet & \bullet & \bullet & T_{1 n} \\
& T_{22} & T_{23} & \bullet & \bullet & \bullet & T_{2 n} \\
& & T_{33} & \bullet & \bullet & \bullet & T_{3 n} \\
& & & \bullet & \bullet & \bullet & \bullet \\
& & & & \bullet & \bullet & \bullet \\
& 0 & & & & \bullet & \bullet \\
& & & & & & T_{n n}
\end{array}\right] .
$$

$\left[T_{i j}\right](i, j=1 \sim n)$ is expressed using matrix $\left[A_{i}\right]$ shown in Eq. $(6 \mathrm{~b})$ as

$$
\left[T_{i j}\right]=\left[A_{i}\right]\left[A_{j}\right]^{T} \text {. }
$$

$\left[\Lambda^{n}\right]$ is a matrix expressed as

where

$$
\left[\Lambda^{n}\right]=\left[\begin{array}{lllll}
\Lambda_{1} & & & \\
& \Lambda_{2} & & 0 \\
& & \Lambda_{3} & \\
& & & \\
& & & \\
& 0 & & & \\
& & & & \Lambda_{n}
\end{array}\right],
$$

$$
\left[\Lambda_{i}\right]=\left[\begin{array}{llllll}
0 & l_{i} & 0 & 1 & 0 & 0 \\
l_{i} & 0 & 0 & 0 & 1 & 0 \\
0 & 0 & 0 & 0 & 0 & 1
\end{array}\right] .
$$


Consequently, joint torques can be obtained, as shown in Eq. (2), by first converting the nodal forces in global coordinates to elemental coordinates and then by multiplying the matrix regarding link lengths.

\subsection{Calculation of incremental nodal forces}

The nodal forces used in Eq. (3) are obtained by solving the following equations of motion:

$$
\begin{gathered}
{[M]\{\ddot{u}\}_{t+\Delta t}=\{F\}_{t+\Delta t},} \\
\{\ddot{u}\}_{t+\Delta t}=\{\ddot{u}\}_{t}+\{\Delta \ddot{u}\},
\end{gathered}
$$

where $[M]$ is the total mass matrix, $\{F\}$ is the external force vector, and $\{\ddot{u}\}$ is the acceleration vector. By adopting Newmark's $\beta$ method (Newmark, 1959) as the time integration scheme and substituting the equation

$$
\{F\}_{t+\Delta t}=\{F\}_{t}+\{\Delta f\}
$$

into Eq. (12), one can derive the following equation for calculating the incremental nodal force vector:

$$
\{\Delta f\}=-\{F\}_{t}+[M]\left(\frac{1}{\beta \Delta t^{2}}\{\Delta u\}-\frac{1}{\beta \Delta t}\{\dot{u}\}_{t}-\left(\frac{1}{2 \beta}-1\right)\{\ddot{u}\}_{t}\right),
$$

where $\{\Delta u\}$ is the incremental displacement vector and $\{\dot{u}\}$ is the velocity vector. The operation distance between each incremental step calculated from a target trajectory is used as an input for $\{\Delta u\}$. The velocity and acceleration vectors can also be directly substituted as input data; however, we used Newmark's $\beta$ method $(\delta=1 / 2, \beta=1 / 4)$ for calculating the values in the actual program. Each corresponding term in the incremental nodal force vector is then substituted into Eq. (3) to constitute the vector $\left\{P^{n}\right\}$.

\section{Calculation of target motions and estimation of inverse dynamics}

\subsection{Rigid body and flexible models}

In the inverse dynamics calculation using the parallel solution scheme, accurate target motions that compensate for the inertial forces acting at the links and the stiffnesses of the links are required. Maintaining such accuracy is inevitable, especially for flexible models where elastic deformations occurring in link members greatly affect the torque values (Bayo, 1987; Asada et al., 1990; Shabana, 1990; Theodore \& Ghosal, 1995). Moreover, the elastic vibration that occurs in the links becomes a serious issue for the control. Therefore, much effort has been made to model and describe robot dynamics with elastic links and to control them (Cetinkunt \& Yu, 1991; Tzes \& Yurkovich, 1991; Hui et al., 2002; Subudhi \& Morris, 2002; Theodore \& Ghosal, 2003; Wang \& Mills, 2005; Feliu et al., 2006). In contrast, we decided to develop a solution scheme of kinematics using the finite element approach combined with the parallel solution scheme to handle analysed models comprehensively in a single calculation process (Isobe \& Kato, 2008).

The following equation of motion can be used for rigid body models, where the occurrence of elastic deformations and internal forces can be neglected: 


$$
[M]\left\{\ddot{u}_{m}\right\}_{t+\Delta t}=\{F\}_{t+\Delta t} .
$$

Here, $\left\{\ddot{u}_{m}\right\}$ is the acceleration vector for the overall system motion components at time $t+\Delta t$. For flexible models, the components for material deformations and internal forces should be considered and the following equation of motion at time $t+\Delta t$ is adopted (Bathe, 1996):

$$
[M]\left\{\ddot{u}_{m}\right\}_{t+\Delta t}+[M]\left\{\ddot{u}_{d}\right\}_{t+\Delta t}+[C]\left\{\dot{u}_{d}\right\}_{t+\Delta t}+[K]\left\{\Delta u_{d}\right\}=\{F\}_{t+\Delta t}-\{R\}_{t},
$$

where $[C]$ is the total damping matrix, $[K]$ is the total stiffness matrix, and $\{R\}$ is the internal force vector. The vectors $\left\{\ddot{u}_{d}\right\},\left\{\dot{u}_{d}\right\}$ and $\left\{\Delta u_{d}\right\}$ denote the acceleration vector, the velocity vector, and the incremental displacement vector for the material deformation components at time $t+\Delta t$, respectively. By applying Newmark's $\beta$ method $(\delta=1 / 2)$ as a time integration scheme, the acceleration and velocity vectors are calculated as

$$
\begin{gathered}
\left\{\ddot{u}_{m}\right\}_{t+\Delta t}=\frac{1}{\beta \Delta t^{2}}\left\{\Delta u_{m}\right\}-\frac{1}{\beta \Delta t}\left\{\dot{u}_{m}\right\}_{t}-\left(\frac{1}{2 \beta}-1\right)\left\{\ddot{u}_{m}\right\}_{t}, \\
\left\{\ddot{u}_{d}\right\}_{t+\Delta t}=\frac{1}{\beta \Delta t^{2}}\left\{\Delta u_{d}\right\}-\frac{1}{\beta \Delta t}\left\{\dot{u}_{d}\right\}_{t}-\left(\frac{1}{2 \beta}-1\right)\left\{\ddot{u}_{d}\right\}_{t}, \\
\left\{\dot{u}_{m}\right\}_{t+\Delta t}=\frac{1}{2 \beta \Delta t}\left\{\Delta u_{m}\right\}-\left(\frac{1}{2 \beta}-1\right)\left\{\dot{u}_{m}\right\}_{t}-\left(\frac{1-4 \beta}{4 \beta}\right)\left\{\ddot{u}_{m}\right\}_{t} \Delta t, \\
\left\{\dot{u}_{d}\right\}_{t+\Delta t}=\frac{1}{2 \beta \Delta t}\left\{\Delta u_{d}\right\}-\left(\frac{1}{2 \beta}-1\right)\left\{\dot{u}_{d}\right\}_{t}-\left(\frac{1-4 \beta}{4 \beta}\right)\left\{\ddot{u}_{d}\right\}_{t} \Delta t,
\end{gathered}
$$

where $\left\{\dot{u}_{m}\right\}$ is the velocity vector for the overall system motion components. Substituting Eqs. (18) into Eq. (17) yields

$$
\begin{aligned}
& \left([K]+\frac{1}{\beta \Delta t^{2}}[M]+\frac{1}{2 \beta \Delta t}[C]\right)\left\{\Delta u_{d}\right\}=\{F\}_{t+\Delta t}-\{R\}_{t}+[M]\left(\frac{1}{\beta \Delta t}\left\{\dot{u}_{d}\right\}_{t}+\left(\frac{1}{2 \beta}-1\right)\left\{\ddot{u}_{d}\right\}_{t}\right) \\
& -[M]\left(\frac{1}{\beta \Delta t^{2}}\left\{\Delta u_{m}\right\}-\frac{1}{\beta \Delta t}\left\{\dot{u}_{m}\right\}_{t}-\left(\frac{1}{2 \beta}-1\right)\left\{\ddot{u}_{m}\right\}_{t}\right)+[C]\left(\left(\frac{1}{2 \beta}-1\right)\left\{\dot{u}_{d}\right\}_{t}+\left(\frac{1-4 \beta}{4 \beta}\right)\left\{\ddot{u}_{d}\right\}_{t} \Delta t\right) .
\end{aligned}
$$

By applying $\left\{\Delta u_{m}\right\}$ as the input in a time-integration loop of Eq. (19) and using the vectors of Eqs. (18) at time $t,\left\{\Delta u_{d}\right\}$ at each time step can be successively obtained. The displacement vector $\left\{u_{m}\right\}$ for the overall system motion components and the displacement vector $\left\{u_{d}\right\}$ for the material deformation components are calculated incrementally as

$$
\begin{gathered}
\left\{u_{m}\right\}_{t+\Delta t}=\left\{u_{m}\right\}_{t}+\left\{\Delta u_{m}\right\}, \\
\left\{u_{d}\right\}_{t+\Delta t}=\left\{u_{d}\right\}_{t}+\left\{\Delta u_{d}\right\} .
\end{gathered}
$$

The total displacement $\{u\}$ is obtained by summing both the overall system motion and the material deformation components as 


$$
\{u\}_{t+\Delta t}=\left\{u_{m}\right\}_{t+\Delta t}+\left\{u_{d}\right\}_{t+\Delta t} .
$$

The final target trajectories considering the effects of stiffness and damping are thus obtained using Eq. (21). The resultant forces acting on the elements can also be calculated using the obtained displacements.

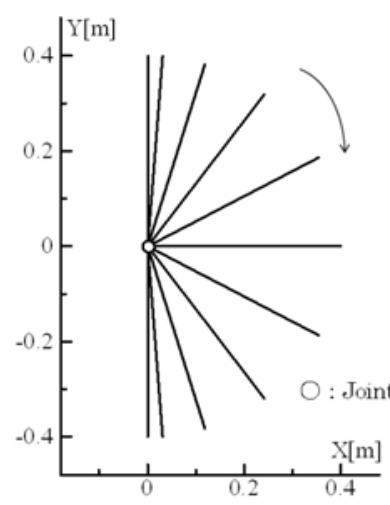

(a)

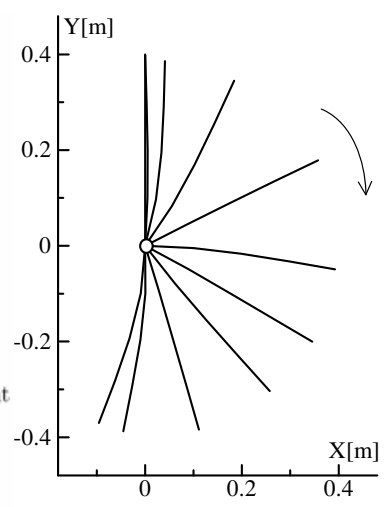

(b)

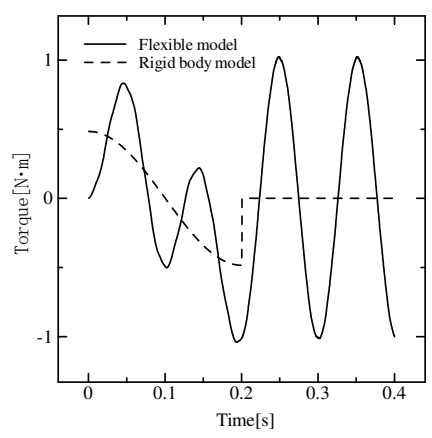

(c)

(a) Initial target motion (target motion for rigid-body model)

(b) Target motion for flexible model obtained by calculating the equation of motion

(c) Torque curves obtained for both models

Fig. 3. Inverse dynamics calculation of a one-joint rigid body/flexible link system

A numerical test was carried out on a one-joint flexible link system to verify the torque values obtained by the calculations. Damping is neglected in the calculations. Figure 3(a) shows a target trajectory of a 0.2-s motion in the horizontal plane for a rigid-body one-link system (link length: $40 \mathrm{~cm}$; weight: $22.4 \mathrm{~g}$; flexural stiffness: perfect rigidity). Using this target motion as input data for the algorithm described above, the motion considering the effect of stiffness is obtained, as shown in Fig. 3(b). Here, the flexural stiffness for the flexible model is given as $0.46 \mathrm{Nm}^{2}$. From the results, we can see the difference between the deformations of the two link systems. Figure 3(c) shows the torque curves for the two models obtained by the parallel solution scheme using the motions of Figs. 3(a) and 3(b) as input data. A smooth joint torque curve is obtained for the rigid-body model. In contrast, a vibration can be observed in the torque curve of the flexible model due to the flexural vibration that occurs in the model, particularly after the motion has stopped. The natural frequency of the model estimated from the torque curve agrees with the theoretical value, which is $10.02 \mathrm{~Hz}$.

\subsection{Underactuated models}

The calculation process described in the previous section can be commonly used for rigid body, flexible, and underactuated models. However, some special treatments should be carried out for the underactuated model due to its large deformation. First, we use an elemental stiffness matrix equipped with only axial components for nonactuated links in order to exclude the transmission of bending moments at the nonactuated joints. However, 
the exclusion of the bending moment effect is not sufficient to realise the behaviour of passive joints because the accumulation of numerical errors due to large deformation occurs in the calculation. Therefore, an algorithm updating the coordinates of the system during time steps is also developed.

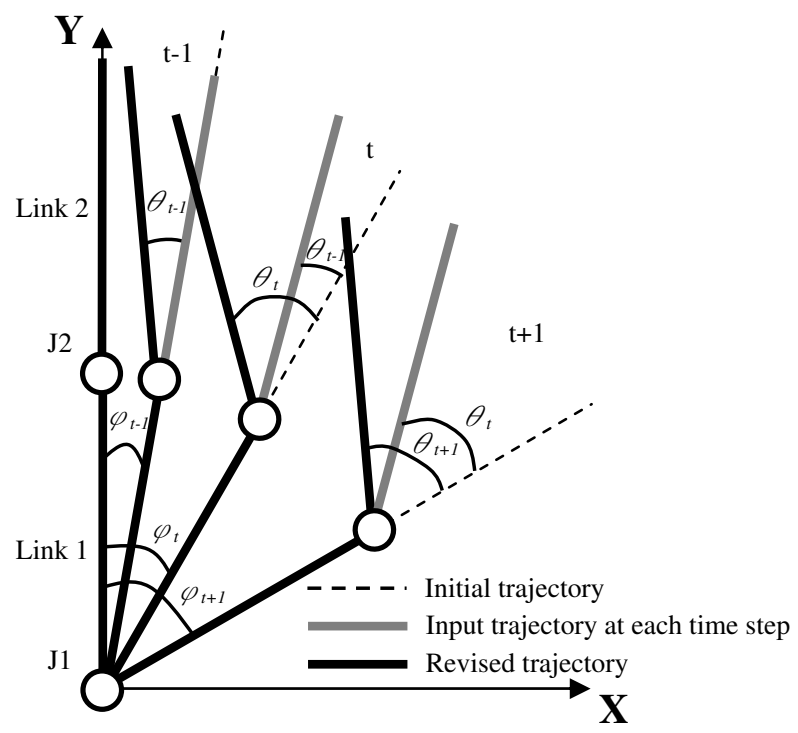

Fig. 4. Outline of updated transformation algorithm used in the calculation of target motions for underactuated models

The outline of the updated transformation algorithm is explained using a simple example of a two-joint underactuated link system as shown in Fig. 4 . The broken lines in the figure are the initial trajectory, the gray lines are the input trajectory at each time step, and the dark lines are the revised trajectories at the end of each time step. $\varphi$ is the rotational angle of the active joint (J1, in this case), which will not be revised by the algorithm, and $\theta$ is the rotational angle of the nonactuated joint (J2, in this case), which will be calculated using the deformation obtained from Eq. (20b). Suppose the posture at time step $t-1$ is represented by the dark line shown in Fig. 4, with the rotational angle of J1 being $\varphi_{\mathrm{t}-1}$. The input trajectory at the next time step $t$ is calculated by only rotating the active joint to $\varphi_{\mathrm{t}}$, whereas the overall posture is fixed as it is. The deformations of the link system at time step $t$ are calculated starting from this posture (gray line at time step $t$ ). The calculated result at the end of time step $t$ will become as the dark line (with the rotational angle of J1 being $\varphi_{\mathrm{t}}$ ), and the revisions proceed successively to the next time step $t+1$ in the same manner. In general $n$-link cases, the input trajectory can be revised by applying the above algorithm from the base joint to the end joint successively at each time step.

The kinematics calculation using the algorithm shown above is carried out for a two-joint underactuated link system. Let the initial target motion of a rigid model be a horizontal 2-s motion, as shown in Fig. 5(a). J1 is an active joint, whereas J2 is a nonactuated joint. The parameters of the links are as follows: material of the links, SUS430; flexural stiffness, 20.84 $\mathrm{Nm}^{2}$; link lengths, $0.25 \mathrm{~m}$; link mass, $9.75 \times 10^{-2} \mathrm{~kg}$; extra mass at the tip, $0.1 \mathrm{~kg}$. The time 


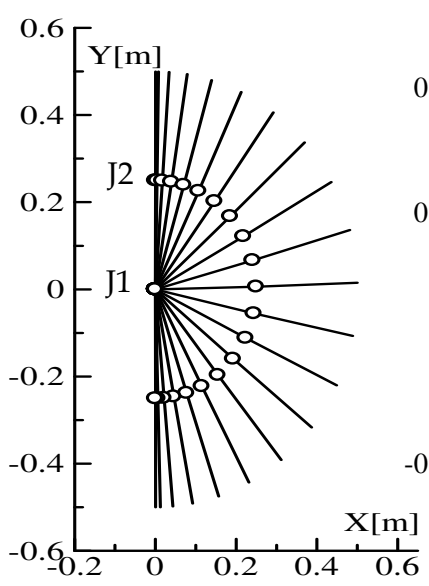

(a)

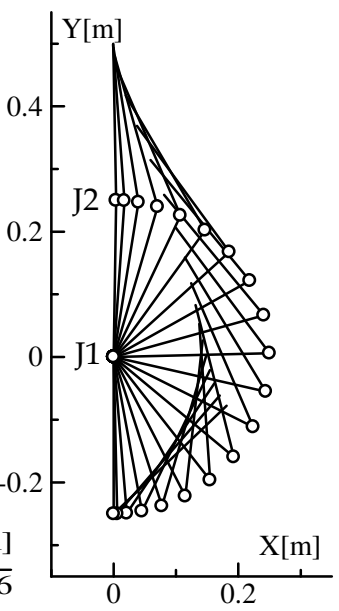

(b)

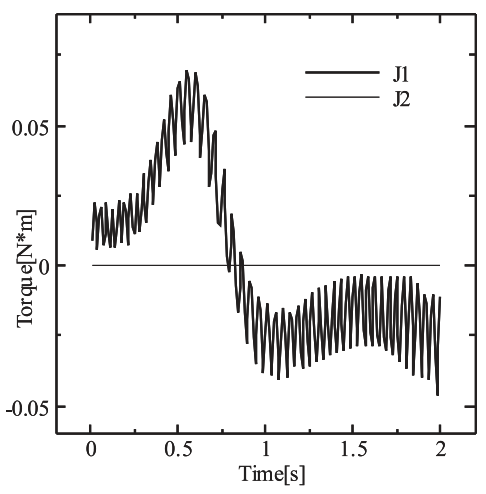

(c)

(a) Initial target motion

(b) Target motion calculated using the updated transformation algorithm

(c) Torque curves of a two-joint underactuated link system obtained using parallel solution scheme

Fig. 5. Inverse dynamics calculation of a two-joint underactuated link system

increment for the calculation is set to $10 \mathrm{~ms}$. Figure 5(b) shows the calculated target motion of the two-joint underactuated model. Figure 5(c) shows the torque curves calculated using the data in Fig. 5(b) as input for the parallel solution scheme. The torque curve for $\mathrm{J} 2$ retains the value of 0 throughout the motion, which is proof that the joint is successfully expressed as a nonactuated joint. The vibration seen in the torque curve for J1 is proof that Link 1 has stiffness (or flexibility). It vibrates with a natural period in the range of $0.025 \mathrm{~s}-0.04 \mathrm{~s}$ (note that the natural period changes with the posture), which matches the theoretical value for a two-link free vibration beam, which is $0.031 \mathrm{~s}$. See the reference (Isobe, 2008) for further numerical examples and experimental results.

\section{Torque Cancelling System (TCS)}

\subsection{General concept of TCS}

A robot swinging around an arm in a quick motion, for example, normally needs a counterbalance motion to avoid any mechanical sway. The TCS proposed in this chapter is a system that generates anti-torque to suppress the mechanical sway by computing accurate inverse dynamics of the arm motion with an accurate consideration of the dynamics of the robot itself. The accurate consideration of the dynamics is carried out by implementing the parallel solution scheme described in the previous sections.

A simple explanation of the concept is provided in Fig. 6 for the uniaxial case. Suppose a rotor is rotated by a motor with a specific torque. A reaction moment will occur around the rotating axis that will make the whole body twist around the axis. To prevent the reaction moment from making the twist, a cancelling moment can be generated by another motor placed on the axis by supplying a specific, cancelling torque. The same concept is used in twin-rotator helicopters, where two rotators rotate in opposite directions to cancel the twist 


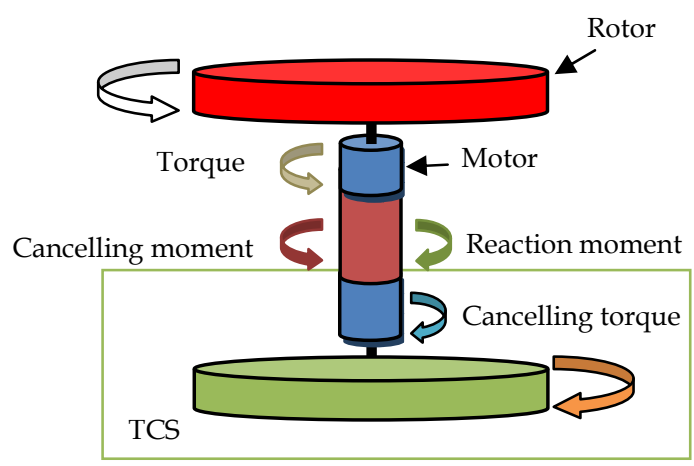

Fig. 6. General concept of uniaxial TCS

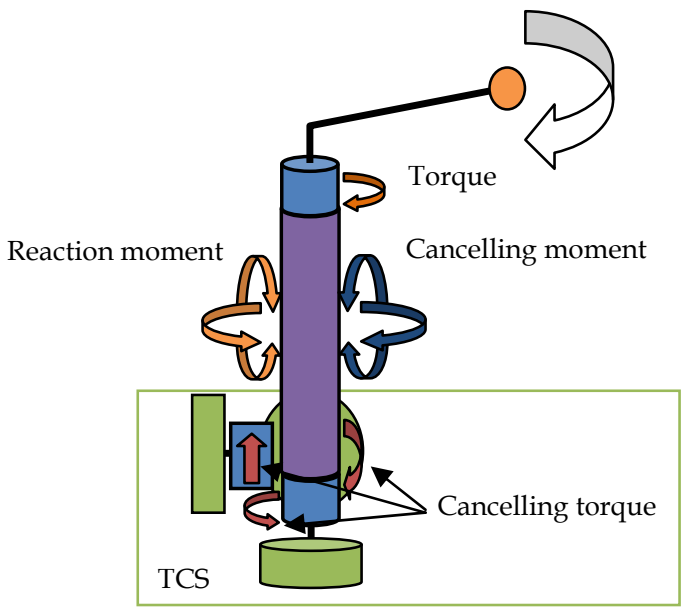

Fig. 7. General concept of triaxial TCS

motion. However, the difference between the twin-rotator helicopters and the proposed TCS is that the twin-rotators only rotate in opposite directions with the same rotational speed, whereas the TCS is driven using accurate torques by considering the dynamics related to the motion and the overall architecture.

A general concept of triaxial TCS is drawn in Fig. 7. If an object in motion follows the model of an outboard rotor (or a cantilever beam as shown in the figure), the reaction moments acting on the body are not as simple as those in the uniaxial case. The moments will act around three-dimensional axes because the centre of gravity of the overall body is at the offset position from the rotating axis. To cancel these moments, three TCSs, each set on each dimensional axis, should be placed as shown in Fig. 7. However, all of the TCSs do not have to be placed at one location or placed exactly on the rotating axis to maintain the function. Instead, a TCS can be mounted, literally, anywhere in the body. As shown later in this chapter, all a TCS has to do to suppress a mechanical sway is to cancel the moment generated at the precise location of the TCS. 


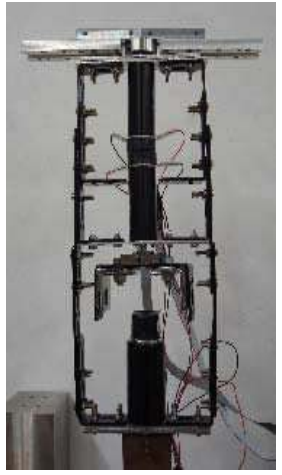

(a)

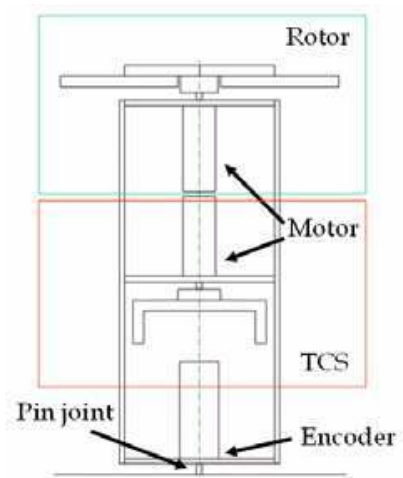

(b)

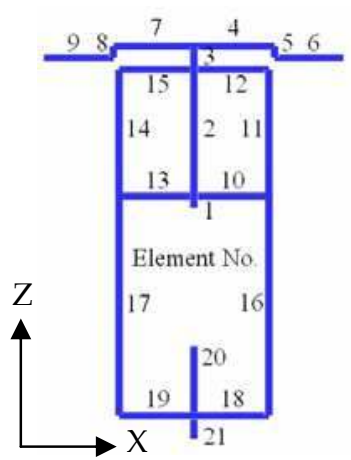

(c)

(a) Experimental setup

(b) Layout of a rotor and a TCS

(c) Finite element subdivision of the setup

Fig. 8. Experimental setup and numerical modelling of a rotor mechanism with uniaxial TCS mounted on the rotating axis

\subsection{Uniaxial TCS mounted on rotating axis to cancel rigid-rotor motion}

A simple rotor-TCS experimental setup, as shown in Figs. 8(a) and 8(b), was made to carry out a numerical estimation and some experiments for verification. In this setup, a rotor is driven by a gearless motor, and a prototype TCS is placed underneath, exactly on the rotating axis of the rotor. The frame body is connected to the ground with a rotation-free pin joint, where an encoder attached to a nonacutated motor is used to detect the rotational angle of the frame body. The rotor is made of aluminium, and the frame body is made of stainless steel, both with enough sectional area such that the whole architecture can be assumed to be rigid without any elastic deformation.

Figure 8(c) shows the finite element subdivision of the experimental setup, with each connection to connection modelled with a single Bernoulli-Euler beam element. The whole system is modelled with a total of 21 elements and 22 nodes. The capabilities of modelling this kind of complex architecture and computing the dynamics are the main features of the parallel solution scheme.

A rotational motion of $4 \Pi \mathrm{rad}$ in $2 \mathrm{~s}$ is given to the rotor. The torque required for the rotor, which is actually computed at the upper node of element No. 3 in Fig. 8(c), is obtained by the parallel solution scheme as shown in Fig. 9(a). Reaction moments generated at the exact location of the TCS (lower node of element No. 1 in Fig. 8(c)) are calculated by the scheme as shown in Fig. 9(b). To cancel these moments, the torque shown in Fig. 9(c) should be supplied to the TCS. Note that the cancelling torque the TCS has to generate is a perfect duplicate of the reaction moment $M_{z}$ (see Fig. 6).

Figure 10 shows the rotational angle of the frame body detected at the encoder attached on the pin joint when the motion torque is actually supplied to the rotor. It can be confirmed from the figure that the frame body is unstable during the motion when the TCS is not activated, whereas the rotation around the pin joint completely stops when the TCS is activated, which means that the reaction moments generated around the rotating axis are perfectly cancelled by supplying the accurately computed cancelling torques to the TCS. 


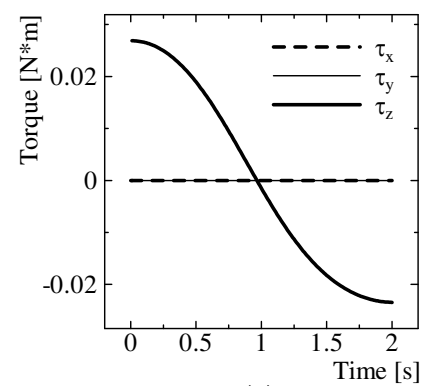

(a)

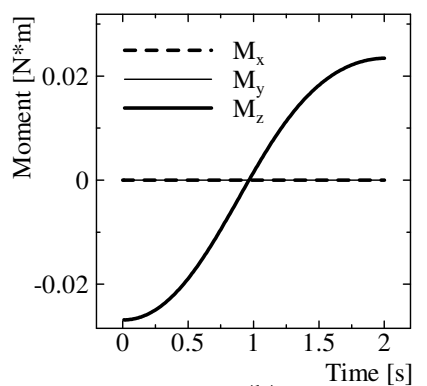

(b)

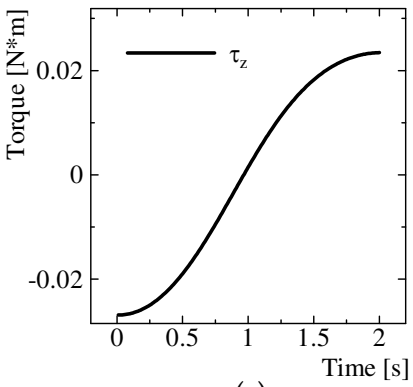

(c)

(a) Motion torques for driving a rotor

(b) Reaction moments generated at the exact location of TCS

(c) Input torque for TCS

Fig. 9. Motion torques, reaction moments, and input torque for uniaxial TCS mounted on the rotating axis

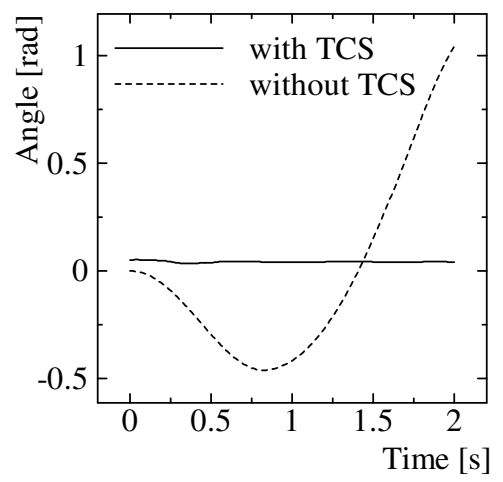

Fig. 10. Rotational angle of the frame body around the pin joint during the motion

\subsection{Uniaxial TCS mounted in offset position to cancel rigid-rotor motion}

Next, an experiment was carried out on a different configuration of the frame body, as shown in Figs. 11(a) and 11(b). In this case, a TCS is mounted in the offset position of the rotating axis to see if such a condition affects the capability of the system. The whole system is modelled with a total of 25 elements and 26 nodes as shown in Fig. 11(c). The same rotational motion is given to the rotor as in the previous experiment, and, consequently, there is no difference between Fig. 9(a) and Fig. 12(a). The motion torques for driving the rotor are the values calculated at the upper node of element No. 3, and the reaction moments are the values calculated at the upper node of element No. 25. The reaction moment and the input torque for the TCS around the $z$ axis in Figs. 12(b) and 12(c) perfectly agree with those in Figs. 9(b) and 9(c). On the other hand, $M_{y}$ in Fig. 12(b) is constantly shifted to the positive direction because a moment due to gravity subjected along the offset length is now generated. The moment generated by gravity, however, is supported at the pin joint and is not meant to be suppressed in this experiment. 


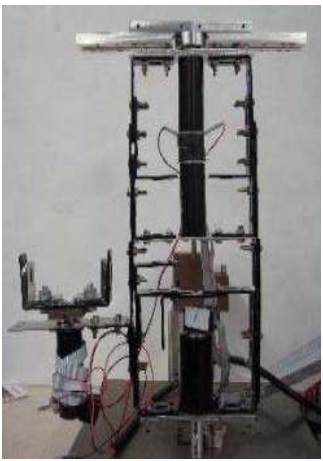

(a)

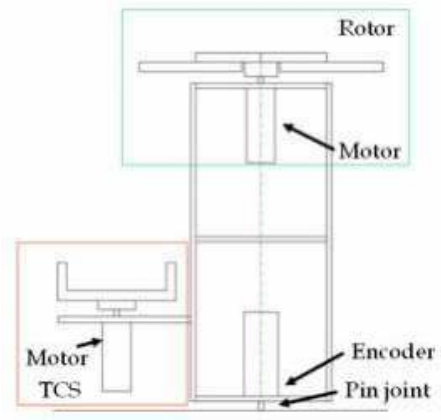

(b)

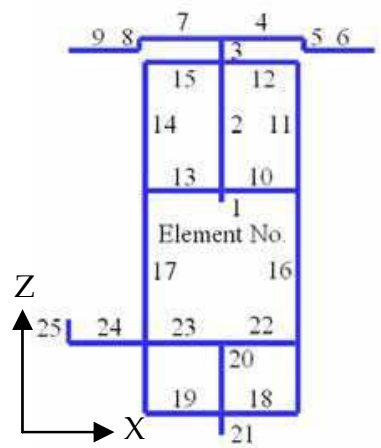

(c)

(a) Experimental setup

(b) Layout of a rotor and a TCS

(c) Finite element subdivision of the setup

Fig. 11. Experimental setup and numerical modelling of a rotor mechanism with uniaxial TCS mounted in the offset position of the rotating axis

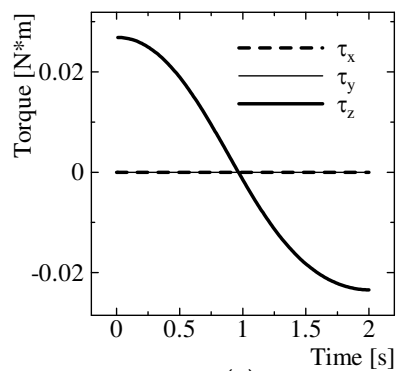

(a)

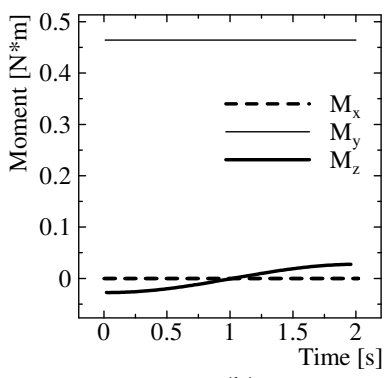

(b)

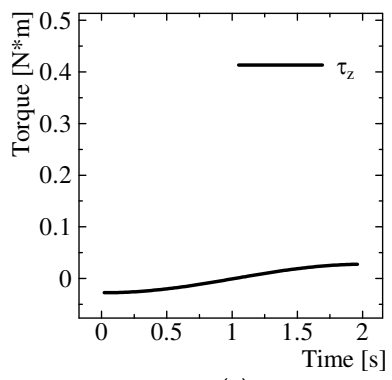

(c)

(a) Motion torques for driving a rotor

(b) Reaction moments generated at the exact location of TCS

(c) Input torque for TCS

Fig. 12 Motion torques, reaction moments, and input torque for uniaxial TCS mounted in the offset position 
It can be confirmed from Fig. 13 that in this case, the mechanical sway around the pin joint is also successfully suppressed to a minimal amount by activating the TCS mounted in the offset position.

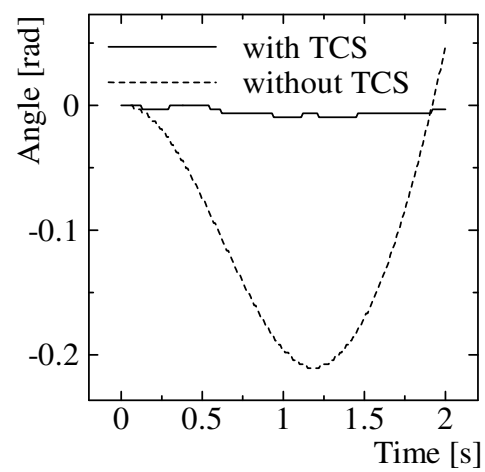

Fig. 13. Rotational angle of the frame body around the pin joint during the motion when a TCS is mounted in the offset position

\subsection{Uniaxial TCS mounted in offset position to cancel flexible-link motion}

Furthermore, a simple flexible link with TCS experimental setup, as shown in Figs. 14(a) and 14(b), was made to carry out a numerical estimation and some experiments for verification. In this setup, a flexible link is driven by a gearless motor, and a prototype TCS is placed at an offset position from the rotating axis of the active joint. The link system is connected to the ground with a rotation-free passive joint, where an encoder attached to a nonacutated motor is used to detect the rotational angle of the overall body. The flexible link is made of poly-carbonate, and the elastic deformation occurring in the link should not be neglected in the inverse dynamics calculation. The experimental setup is subdivided, with each connection to connection represented by a single Bernoulli-Euler beam element. The whole system is modelled with a total of 4 elements and 5 nodes as shown in Fig. 14(c). The capabilities of modelling this kind of architecture with flexible link and computing the dynamics are, again, the main features of the parallel solution scheme.

A rotational motion of $\Pi$ rad in $2 \mathrm{~s}$ is given to the active link. The torque required for the active joint is obtained by the parallel solution scheme as shown in Fig. 15(a). The reaction moment generated at the exact location of the TCS is calculated as shown in Fig. 15(b). To cancel this moment, the torque shown in Fig. 15(c) should be supplied to the TCS. Here again, note that the cancelling torque that the TCS must generate is a perfect duplicate of the reaction moment (see Fig. 6).

Figure 16 shows the rotational angle of the link system detected at the encoder attached on the passive joint when the motion torque is actually supplied to the active joint. It can be confirmed from the figure that the link system is unstable during the motion when the TCS is not activated, whereas the rotation around the passive joint completely stops when the TCS is activated, which means that the vibrating reaction moment generated around the rotating axis is perfectly cancelled by supplying the accurately computed cancelling torque to the TCS. 


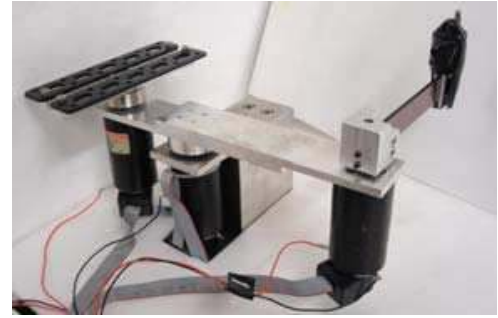

(a)

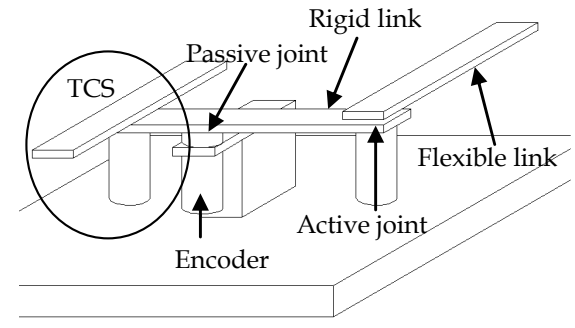

(b)

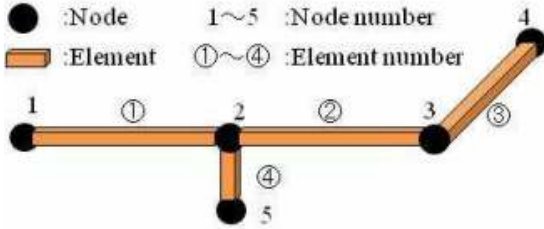

(c)

(a) Experimental setup

(b) Configuration of the setup and location of a TCS

(c) Finite element subdivision of the setup

Fig. 14. Experimental setup and numerical modelling of a flexible link mechanism with uniaxial TCS mounted in the offset position of the rotating axis

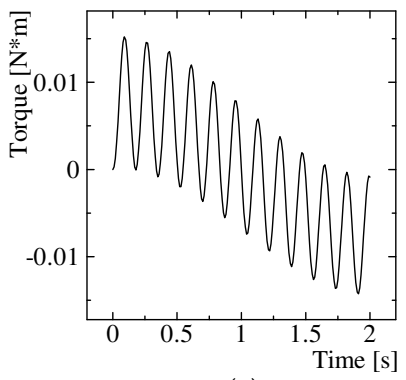

(a)

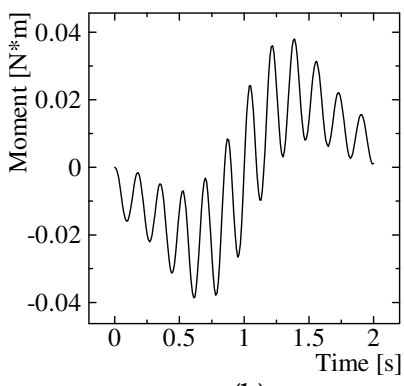

(b)

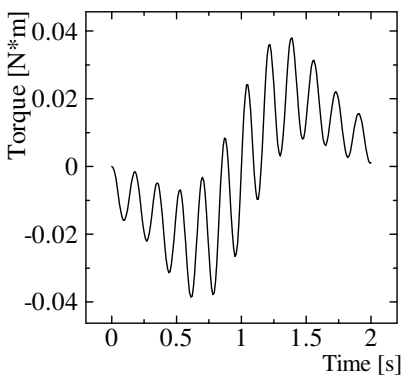

(c)

(a) Motion torque for driving the flexible link

(b) Reaction moment generated at the exact location of TCS

(c) Input torque for TCS

Fig. 15. Input torques and generated moments in the system 


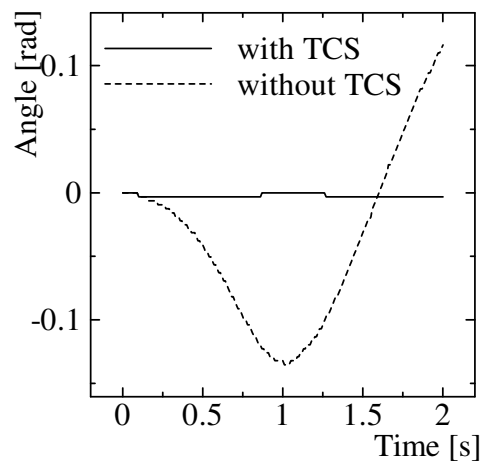

Fig. 16. Rotational angle of the link system around the passive joint during the motion

\section{Application of TCS on a Robot}

The TCS shown in this chapter can be applied to actual robotic architecture to suppress mechanical sway generated by rotational motions. A sample of a walking robot mounted with a TCS, along with its configuration and finite element subdivision, are shown in Fig. 17. A TCS shown in Fig. 17(a) is mounted at the central part of the robot, as shown in Fig. 17(b). As shown in Fig. 17(c), a caster at the central-bottom part supports the overall weight of the robot, while two electric magnet holders at the tip of two legs are used to get hold of a metallic base. Two motors (Motor 1 and Motor 2 in Fig. 17(c)) are activated to advance the steps, and the other two motors on the legs are used to lift up and lower the legs. Mechanical sway is often generated during the motions of Motor 1 and Motor 2 because the architecture possesses a comparatively large inertia. A TCS that is mounted at the central part of the body can be applied to suppress the sway during the gait motion. The robot is modelled numerically, as shown in Fig. 17(d), with finite elements and applied to the calculation of various required torques. A two-step gait for the walking robot is given as shown in Fig. 18. The motion torques for the two motors (Motor 1 and Motor 2) along with the input torque for the TCS during the motion is computed using the scheme described previously.

The calculated motion torques for the motors and input torque for the TCS are shown in Fig. 19. Large torques are required during the walking step motions at $1.0 \mathrm{~s} \sim 1.5 \mathrm{~s}$ and $3.5 \mathrm{~s} \sim$ $4.25 \mathrm{~s}$ (see Fig. 19(a) and 19(b)), and reaction moments are generated during those periods at various parts of the robotic architecture. The reaction moment generated at the exact location of the TCS is calculated by the parallel solution scheme as shown in Fig. 19(c), which is used directly as an input torque for the TCS. Some experiments using the setup are scheduled in the near future, and the latest results will be reported on our website (http://www.kz.tsukuba.ac.jp/ isobe/) and in technical papers. 


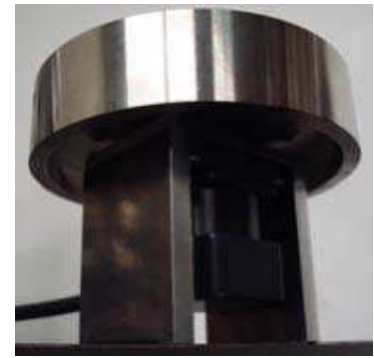

(a)

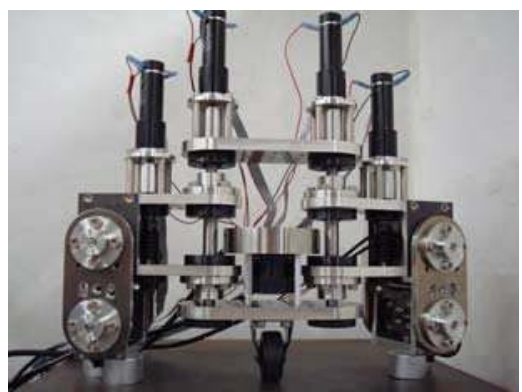

(b)

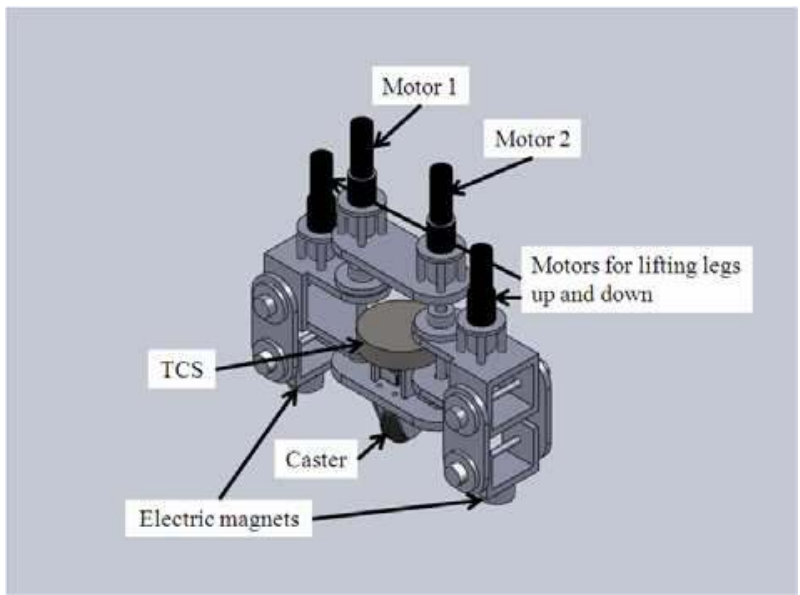

(c)

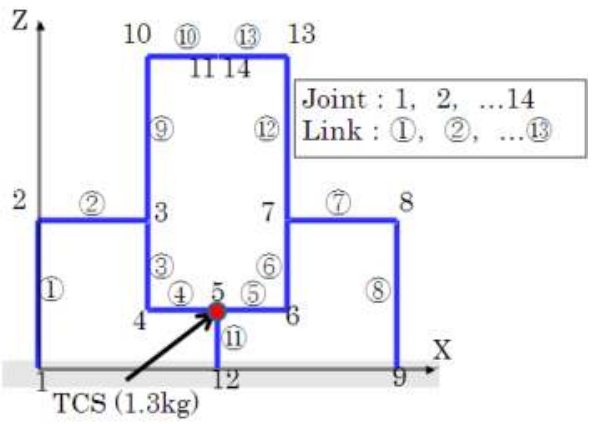

(d)

(a) View of a TCS mounted in a robot

(b) General view (TCS mounted at the central part)

(c) Configuration of the robot and location of the TCS

(d) Finite element subdivision of the robot

Fig. 17. A walking robot mounted with a TCS 


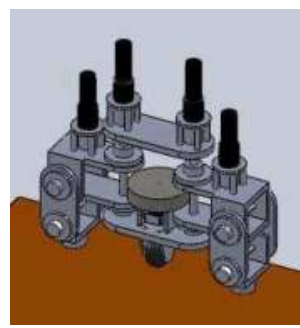

(a)

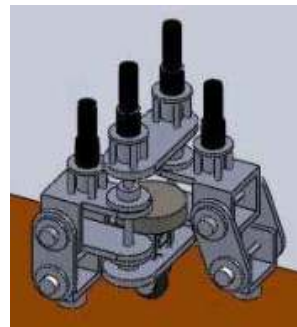

(e)

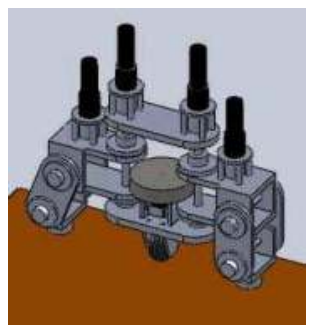

(b)

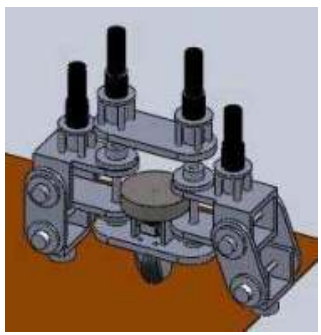

(f)

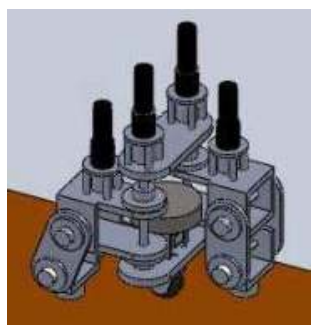

(c)

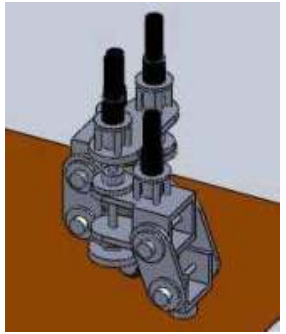

(g)

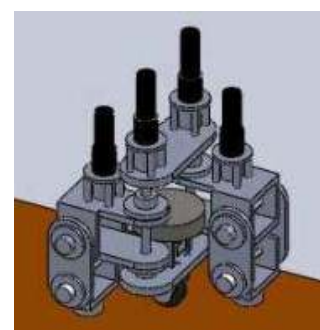

(d)

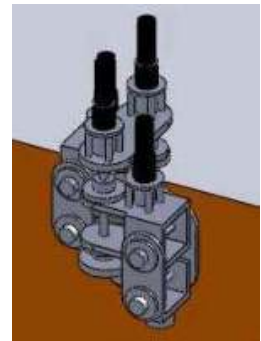

(h)
(a) Initial position $(0.0 \mathrm{~s})$
(b) Lift up the right leg $(0.0 \mathrm{~s} \sim 1.0 \mathrm{~s})$
(c) First step motion $(1.0 \mathrm{~s} \sim 1.5 \mathrm{~s})$
(d) Lower the right leg $(1.5 \mathrm{~s} \sim 2.5 \mathrm{~s})$
(e) Lift up the left leg $(2.5 \mathrm{~s} \sim 3.5 \mathrm{~s})$
(f) (g) Second step motion (3.5 s $\sim 4.25 \mathrm{~s}$ )
(h) Lower the left leg $(4.25 \mathrm{~s} \sim 5.25 \mathrm{~s})$

Fig. 18. A two-step gait for the walking robot

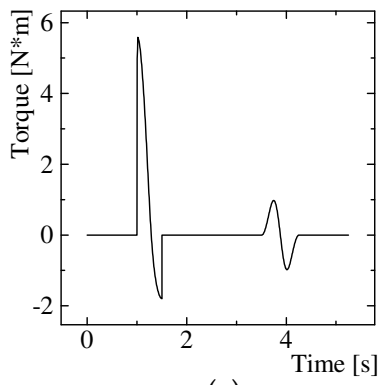

(a)

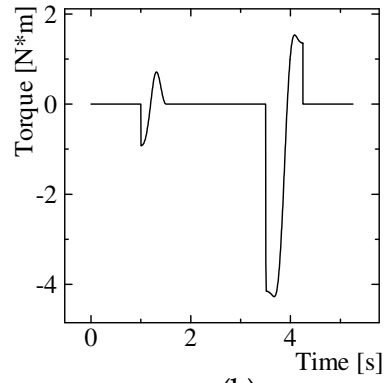

(b)

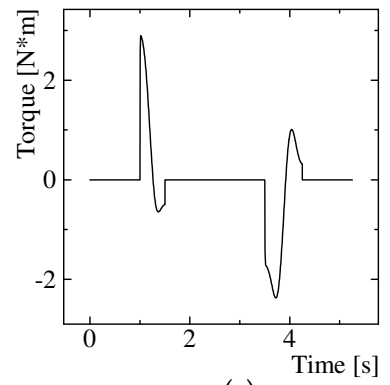

(c)

(a) Motion torque for Motor 1

(b) Motion torque for Motor 2

(c) Reaction moment generated at the exact location of TCS (or input torque for TCS)

Fig. 19. Motion torques for the motors and input torque for the TCS 


\section{Conclusion}

Modelling the dynamics of a robotic architecture usually requires ambiguous assumptions and labour-intensive formulations at various stages. However, application of the finite element approach to inverse dynamics calculation enables us to consider the precise dynamics of robots using a very simple process. This approach leads us to simpler and more precise feedforward control of robots as well as the cancellation of mechanical sway during quick motions. We are now attempting to develop a compact TCS device that can be easily mounted on actual robots in both uniaxial and triaxial cases.

\section{Acknowledgment}

The author wishes to acknowledge the contributions of the following former and current graduate students of his laboratory: Takeo Ueda, Daisaku Imaizumi, Youichi Chikugo, Shunsuke Sato, Atsushi Yagi, Akihiro Kato, Koji Yamanaka, Hiromasa Ueda, Yuto Kitamura, Junya Hayakawa, Yasumasa Matsui, Kensuke Kondo, and Bumpei Nakayama.

\section{References}

Asada, H.; Ma, Z.D. \& Tokumaru, H. (1990). Inverse Dynamics of Flexible Robot Arms: Modelling and Computation for Trajectory Control, ASME J. Dyn. Sys. Measurement Control, Vol. 112, pp. 177-185.

Bathe, K.J. (1996). Finite Element Procedures, Prentice-Hall.

Bayo, E. (1987). A Finite-Element Approach to Control the End-Point Motion of a SingleLink Flexible Robot, J. Robotic Systems, Vol. 4, No. 1, pp. 63-75.

Cetinkunt, S. \& Yu, W.L. (1991). Closed Loop Behavior of a Feedback Controlled Fexible Arm: a Comparative Study, Int. J. of Robotics Research, Vol. 10, No. 3, pp. 263-275.

Feliu, V.; Pereira, E.; Díaz, I.M. \& Roncero, P. (2006). Feedforward Control of Multimode Single-Link Flexible Manipulators based on an Optimal Mechanical Design, Robotics and Autonomous Systems, Vol. 54, No.8, pp. 651-666.

Hui, D.; Fuchun, S. \& Zengqi, S. (2002). Observer-Based Adaptive Controller Design of Flexible Manipulators using Time-Delay Neurofuzzy Networks, J. of Intelligent and Robotic Systems, Vol. 34, No. 4, pp. 453-456.

Isobe, D.; Takeuchi, H. \& Ueda, T. (2000). A Finite-Element Approach to Control Link Mechanisms: Its Concept and Basic Simulation, Proc. Int. Conf. Computational Engineering Science 2000 -Advances in Computational Engineering and Sciences-, pp. 1648-1653, Los Angeles, USA.

Isobe, D.; Imaizumi, D.; Chikugo, Y. \& Sato, S. (2003). A Parallel Solution Scheme for Inverse Dynamics and Its Application in Feed-forward Control of Link Mechanisms, J. of Robotics and Mechatronics, Vol. 15, No. 1, pp. 1-7.

Isobe, D. (2004). A Unified Solution Scheme for Inverse Dynamics, Advanced Robotics, Vol. 18, No. 9, pp. 859-880.

Isobe, D.; Yagi, A. \& Sato, S. (2006). General-Purpose Expression of Structural Connectivity in the Parallel Solution Scheme and Its Application, JSME Int. J. Series C, Vol. 49, No. 3, pp. 789-798.

Isobe, D. \& Kato, A. (2008). Feedforward Control of Flexible Link Systems using Parallel Solution Scheme, Int. J. Robotics and Automation, Vol.23, No.1, pp.31-39. 
Isobe, D. (2008). Unified Solution Scheme for Inverse Dynamics of Robotic Systems, In: Progress in Autonomous Robot Research, Mortensen, F.N. (Ed.), pp.125-173, Nova Science Publishers, ISBN:978-1-60456-281-1.

Nakamura, Y. \& Ghodoussi, M. (1989). Dynamics Computation of Closed-Link Robot Mechanisms with Nonredundant and Redundant Actuators, IEEE Trans. Robotics and Automation, Vol. 5, No. 3, pp. 294-302.

Nakamura, Y. \& Yamane, K. (2000). Dynamics Computation of Structure-Varying Kinematic Chains and Its Application to Human Figures, IEEE Trans. Robotics and Automation, Vol. 16, No. 2, pp. 124-134.

Newmark, N.M. (1959). A Method of Computation for Structural Dynamics, A.S.C.E. J. Engineering Mechanics, Vol. 85, pp. 67-94.

Ookami, Y.; Tomita, N.; Nakasu, S. \& Matsunaga, S. (2008). Introduction to the Space Station, University of Tokyo Press, pp. 204-208, in Japanese.

Shabana, A.A. (1990). Dynamics of Flexible Bodies Using Generalized Newton-Euler Equations, ASME J. Dyn. Sys. Measurement Control, Vol. 112, pp. 496-503.

Subudhi, B. \& Morris, A.S. (2002). Dynamic Modelling, Simulation and Control of a Manipulator with Flexible Links and Joints, Robotics and Autonomous Systems, Vol. 41, No. 4, pp. 257-270.

Sugimoto, K. (2003). Dynamic Analysis of Closed Loop Mechanisms on the Basis Vectors of Passive Joint Axes, J. Robotic Systems, Vol. 20, No. 8, pp. 501-508.

Theodore, R.J. \& Ghosal, A. (1995). Comparison of the Assumed Modes and Finite Element Models for Flexible Multilink Manipulators, Int. J. Robotics Research, Vol. 14, No. 2, pp. 91-111.

Theodore, R.J. \& Ghosal, A. (2003). Robust Control of Multilink Flexible Manipulators, Mechanism and Machine Theory, Vol. 38, No. 4, pp. 367-377.

Tzes, A.P. \& Yurkovich, S. (1991). Application and Comparison of On-Line Identification Methods for Flexible Manipulator Control, Int. J. of Robotics Research, Vol. 10, No. 5, pp. 515-527.

Wang, X. \& Mills, J.K. (2005). FEM Dynamic Model for Active Vibration Control of Flexible Linkages and Its Application to a Planar Parallel Manipulator, Applied Acoustics, Vol. 66, No. 10, pp. 1151-1161.

Yoshida, K.; Nenchev, D.N. \& Hashizume, K. (2002). Flight Experiments of ETS-VII for Advanced Space Robot Control, J. Society of Japanese Aerospace Companies, Vol. 50, No. 584, pp. 351-359, in Japanese. 


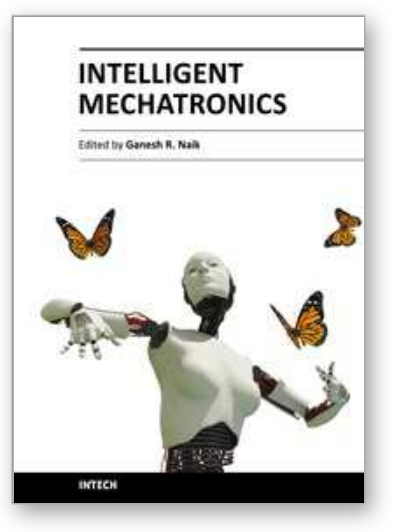

\author{
Intelligent Mechatronics \\ Edited by Prof. Ganesh Naik
}

ISBN 978-953-307-300-2

Hard cover, 248 pages

Publisher InTech

Published online 28, February, 2011

Published in print edition February, 2011

This book is intended for both mechanical and electronics engineers (researchers and graduate students) who wish to get some training in smart electronics devices embedded in mechanical systems. The book is partly a textbook and partly a monograph. It is a textbook as it provides a focused interdisciplinary experience for undergraduates that encompass important elements from traditional courses as well as contemporary developments in Mechtronics. It is simultaneously a monograph because it presents several new results and ideas and further developments and explanation of existing algorithms which are brought together and published in the book for the first time.

\title{
How to reference
}

In order to correctly reference this scholarly work, feel free to copy and paste the following:

Daigoro Isobe (2011). A Torque Cancelling System for Quick-Motion Robots, Intelligent Mechatronics, Prof. Ganesh Naik (Ed.), ISBN: 978-953-307-300-2, InTech, Available from:

http://www.intechopen.com/books/intelligent-mechatronics/a-torque-cancelling-system-for-quick-motion-robots

\section{INTECH}

open science | open minds

\section{InTech Europe}

University Campus STeP Ri

Slavka Krautzeka 83/A

51000 Rijeka, Croatia

Phone: +385 (51) 770447

Fax: +385 (51) 686166

www.intechopen.com

\section{InTech China}

Unit 405, Office Block, Hotel Equatorial Shanghai

No.65, Yan An Road (West), Shanghai, 200040, China

中国上海市延安西路 65 号上海国际贵都大饭店办公楼 405 单元

Phone: +86-21-62489820

Fax: +86-21-62489821 
(C) 2011 The Author(s). Licensee IntechOpen. This chapter is distributed under the terms of the Creative Commons Attribution-NonCommercialShareAlike-3.0 License, which permits use, distribution and reproduction for non-commercial purposes, provided the original is properly cited and derivative works building on this content are distributed under the same license. 